\title{
ASYMPTOTICS OF SMALL EIGENVALUES OF RIEMANN SURFACES
}

\author{
MARC BURGER
}

Recently there has been a great deal of interest in geometric bounds on small eigenvalues of the Laplace operator on a Riemann surface [S.W.Y, D.P.R.S.]. Here we determine the precise asymptotic behaviour of these small eigenvalues. Let $S_{\delta}$ be a compact Riemann surface of genus $g \geq 2$ whose first $k$ nonzero eigenvalues $0<\lambda_{1} \leq \lambda_{2} \leq \cdots \leq \lambda_{k}$ are small, i.e., $\lambda_{k} \leq \delta$ and $\lambda_{k+1} \geq c_{1}$. Then by [S.W.Y.] there exists a constant $a=a(g)>$ 0 such that the closed geodesics $\gamma_{1} \cdots \gamma_{r}$ of length less than $a \cdot \delta$ separate $S_{\delta}$ into $k+1$ pieces $S_{1}, \ldots, S_{k+1}$ and all other closed geodesics of $S_{\delta}$ have length greater than $a(g)$. Let $\Lambda$ be the graph whose vertices are the pieces $S_{i}$. Suppose vertex $S_{i}$ has mass $v_{i}=\operatorname{vol}\left(S_{i}\right)$ and the length $L_{i j}$ of an edge joining $S_{i}$ to $S_{j}$ is the total length of the geodesics contained in $S_{i} \cap S_{j}$. Furthermore, let $0<\lambda_{1}(\Lambda) \leq \cdots \leq \lambda_{k}(\Lambda)$ be the spectrum of the quadratic form $\sum\left(F\left(S_{i}\right)-F\left(S_{j}\right)\right)^{2} L_{i j}$ with respect to the norm $\sum F\left(S_{i}\right)^{2} v_{i}$. Then one has

THEOREM 1.

$$
\lim _{\delta \rightarrow 0} \frac{\lambda_{j}\left(S_{\delta}\right)}{\lambda_{j}(\Lambda)}=\frac{1}{\pi} \quad \text { for all } 1 \leq j \leq k .
$$

This convergence is uniform for all surfaces $S_{\delta}$ with $\lambda_{k+1}\left(S_{\delta}\right) \geq c_{1}$ and fixed genus.

REMARK. The fact that $\lim \sup _{\delta \rightarrow 0} \lambda_{j}\left(S_{\delta}\right) / \lambda_{j}(\Lambda) \leq 1 / \pi$ follows easily from [C.CdV]. This paper also shows the convergence of this ratio in the case that the lengths $l\left(\gamma_{i}\right)$ all have the same behaviour near zero, i.e. $l\left(\gamma_{i}\right)=d_{i} \varepsilon$ for $\varepsilon \rightarrow 0$, and fixed $d_{i}$.

SKETCH OF PROOF. Complete $\gamma_{1} \cdots \gamma_{r}$ to a set of geodesics $\gamma_{1} \cdots \gamma_{3 g-3}$, giving a decomposition of $S$ into $Y$-pieces with length $l\left(\gamma_{i}\right) \leq L_{g}$, a constant depending only on $g$ (see [Bu2, §13]). Then using a modified version of an argument of [B1] we show that $\lambda_{j} \cdot(1+o(\sqrt{\delta})) \geq \pi^{-1} \lambda_{j}(\Gamma)$, where $\Gamma$ is the graph of the $Y$-pieces, and the length of an edge corresponding to a small geodesic is $l(\gamma)$. The proof of this also uses the asymptotic of the first nonzero eigenvalue of $Y_{1} \cup Y_{2}$ for the Neumann problem, where $Y_{1}, Y_{2}$ are $Y$-pieces, $Y_{1} \cap Y_{2}=\gamma$ and $l(\gamma)$ is small. This can be deduced from [C.CdV], because there is only one small geodesic separating $Y_{1} \cup Y_{2}$. To finish the proof we have then to compare $\lambda_{j}(\Gamma)$ with $\lambda_{j}(\Lambda)$. To do this we consider $\Lambda$ to be the graph of the connected components of $\Gamma$ after removing the small edges of $\Gamma$.

Received by the editors February 10, 1987.

1980 Mathematics Subject Classification (1985 Revision). Primary 53C22, 58G25; Secondary 30F99. 
If $f$ is an eigenfunction corresponding to $\lambda_{j}(\Gamma)$ we write $f=h+g$, where, on each component, $h$ is constant and $g$ of mean zero: Applying the quadratic form to $h+g$ we estimate the resulting terms.

If one is interested in lower bounds on $\lambda_{j}$ in terms of $\lambda_{j}(\Lambda)$ it is more convenient to relate $\lambda_{j}$ to a graph associated to a geodesic triangulation of $S$ as considered in [Bu1] and used in [B2, §4]. As in the proof of Theorem 1, one relates then the spectrum of this graph to $\lambda_{j}(\Lambda)$.

THEOREM 2. Let $S$ be a compact Riemann surface of genus $g \geq 2$ and $l\left(\gamma_{1}\right) \leq \cdots \leq l\left(\gamma_{r}\right) \leq l\left(\gamma_{r+1}\right) \cdots$ be the length of the closed geodesics of length smaller than $2 \ln 2$. Then there exists a universal constant $c>0$ s.t. if $l\left(\gamma_{r}\right) \leq l\left(\gamma_{r+1}\right) / g^{2}$ then $\lambda_{j}(S) \geq c \lambda_{j}(\Lambda)$ for $1 \leq j \leq k$. And $\Lambda$ is the graph associated to the components of $S$ after removing the geodesics $\gamma_{1}, \ldots, \gamma_{r}$.

REMARKS. (a) It is easy to generalize Theorems 1 and 2 to the case of geometrically finite surfaces.

(b) In [D.P.R.S.] the inequality of Theorem 2 is stated with a constant $c(g)$ depending on the genus of $S$. But for fixed $g$ there are only finitely many graph structures which can occur if one forgets the length of the edges, thus a constant depending on $g$ "destroys" the combinatorial information contained in $\lambda_{j}(\Lambda)$.

(c) If there are no small geodesics or if the small geodesics don't disconnect the surface then $\lambda_{1}(S) \geq c / g^{2}, c>0$ being a universal constant.

\section{REFERENCES}

[B1] M. Burger, Estimation de petites valeurs propres du Laplacien d'un revêtement de variétés riemanniennes compactes, C.R. Acad. Sci. Paris Sér. I 302 (1986), 191-194.

[B2] _ _ Spectre du Laplacien, graphes et topologie de Fell, preprint.

[Bu1] P. Buser, Cubic graphs and the first eigenvalue of a Riemann surface, Math. Z. 162 (1978), 87-99.

[Bu2] __, Riemannsche Flächen und Längenspektrum vom trigonometrischen Standpunkt aus, Habilitationsschrift, Bonn, 1980.

[C.CdV] B. Colbois and Y. Colin de Verdière, Sur la multiplicité de la première valeur propre d'une surface de Riemann à courbure constante, preprint.

[D.P.R.S.] J. Dodziuk, T. Pignataro, B. Randol, and D. Sullivan, Estimating small eigenvalues of Riemann surfaces, preprint.

[S.W.Y.] R. Schoen, S. Wolpert, and S. T. Yau, Geometric bounds on low eigenvalues of a compact Riemann surface, Geometry of the Laplace Operator, Proc. Sympos. Pure Math., vol. 36, Amer. Math. Soc., Providence, R.I., 1980, pp. 279-285.

MAThematisches Institut, Rheinsprung 21, 4051 BASEL, SWITZERLAND 